\title{
Case Report: Urachal Cyst Presenting In Adult
}

\author{
Authors \\ Dr Rajeev.N. (MS), Dr Santhosh.K. \\ Dr B.R.Ambedkar Medical College \\ Email:drjeevs@gmail.com
}

\begin{abstract}
Introduction: Urachal cyst is very rarely seen in adults. They are normally obliterated in early infancy. Urachal abnormalities are more common in children.

Case Presentation: We describe a case of a 30 year old male who presented with a tender, midline, infraumbilical mass and purulent umbilical discharge. Diagnosis of an infected umbilical sinus was made. He was treated initially with broad spectrum antibiotics, surgery was done after the infection subsided.Histology of the excised fibrous tract showed chronic inflammation with no evidence of malignancy. Postoperative recovery was uneventful.

Conclusion: Urachal abnormalities are rare in adults. Clinical presentation is non-specific, therefore, a high index of suspicion is required in order to make the diagnosis. When diagnosed, surgical excision is advised because of the risk of malignant transformation.
\end{abstract}

\section{Introduction}

Urachus or median umbilical ligament is obliterated allantois. It gets obliterated in fetal life. It extends from the bladder dome to the umbilicus. A partial or total defect in the process of obliteration of the allantois results in urachal abnormalities. They are usually detected in early childhood. They can very rarely present in adulthood. The clinical presentation is variable and makes the diagnosis difficult. In adults, urachal cyst (UC) is the commonest variety, with infection being the usual mode of presentation. Diagnosis remains challenging due to the rarity of this lesion and the non-specific nature of its symptomatology. Since the first description of urachal abnormality by Cabriolus in 1550, few cases have been reported in literature.

\section{Case Presentation}

A 30 year old male was referred after presenting to the emergency department with a 1 month history of persistent purulent umbilical discharge associated with constant lower abdominal pain, chills and rigors. He gave no history of nausea, vomiting or change in bowel habit. Systemic review revealed no abnormality.

On examination, his temperature was 100 degree $\mathrm{F}$ and he was haemodynamically stable. Abdominal examination, showed purulent umbilical discharge with surrounding erythema and a tender infraumbilical mass.

Haematology showed a raised WBC 14,500 with neutrophilia, 84\%. Abdominal ultrasound scan showed a $1.1^{*} 1.4 \mathrm{~cm}$ echogenic area in the umbilical region with surrounding cutaneous edema-umbilical abscess with granulation tissue. 
The patient was initially treated with intravenous antibiotics for 5 days the inflammation subsided and was planned for surgery.

Elliptical incision was placed around the umbilicus and the firrous tract was explored it was traced to the urinary bladder and was excised flush with bladder. The specimen was sent for HPE.

Histopathological examination of the specimen showed umbilical sinus with secondary inflammation with no evidence of malignancy. Recovery was unremarkable.
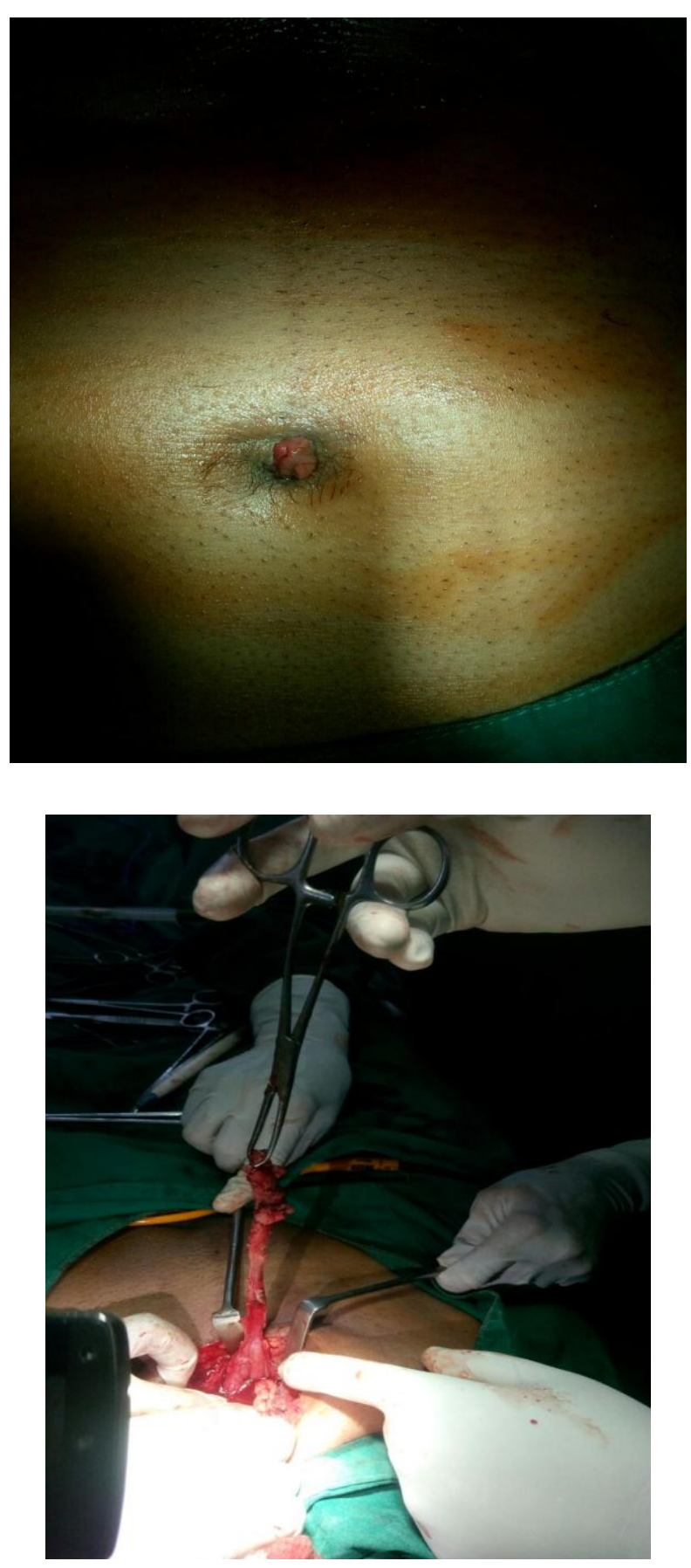

\section{Discussion}

The urachus, developmentally is the upper part of the bladder, both of which arise from the ventral part of the cloaca. Descent of the bladder from the 5 th month of development into the foetal pelvis pulls the urachus with it resulting in the formation of the urachal canal. The lumen of this canal progressively obliterates during foetal life, with eventual formation of a fibrous tract in early adult life. At the end of development, the urachus lies between the transversalis fascia anteriorly and the peritoneum posteriorly (space of Retzius), surrounded by loose areolar tissue and attaches the umbilicus to the dome of the bladder. Histologically, it is composed of 3 layers; an innermost layer of modified transitional epithelium similar to the urothelium, the middle layer of fibroconnective tissue and outermost layer of smooth muscle continuous with the detrusor.

There are five types of urachal abnormalities:

1. patent urachus, in which the entire tubular structure fails to close;

2. urachal cyst, in which both ends of the canal close leaving an open central portion;

3. urachal sinus, which drains proximally into the umbilicus;

4. vesico-urachal diverticulum, where the distal communication to the bladder persists;

5. alternating sinus, which can drain to either bladder or umbilicus.

The incidence of UC in adults is unknown but it is rare. It is more common in men than women. In a 31-year review, Risher et al ${ }^{[2]}$ found 12 adults with urachal anomalies, of which 5 were UC. Modes of presentation of urachal anomalies in adults differ from those seen in children. In adults, the commonest variety is urachal cyst, with infection being usual mode of presentation. The route of infection is haematogenous, lymphatic, direct or ascending from the bladder. The commonly cultured microorganisms from the cystic fluid include Escherichia coli, Enterococcus 
faecium, Klebsiella pneumonia, Proteus, Streptococcus viridans and Fusobacterium,

The clinical signs and symptoms are non-specific, as UC are largely asymptomatic until they become infected. However, the presence of the triad of symptoms including a tender midline infraumbilical mass, umbilical discharge and sepsis should arouse suspicion of UC. If left untreated, UC slowly enlarges and may drain through the umbilicus as was seen in our patient, or drain into the bladder or both, resulting in alternating sinus.

Ultrasound scan can help to make diagnosis in $77 \%$ of patients.

UC can be complicated by rupture into the peritoneal cavity leading to peritonitis. Other reported complications include uracho-colonic fistula, stone formation and neoplastic transformation. The risk of urachal malignancy in adults is high and the prognosis is poor. 54 year retrospective study of 130 adults with urachal abnormalities found that $51 \%$ were malignant and $20 \%$ presented with metastatic disease. The median age at presentation was 61 years and the 2 common risk factors for malignancy were age and haematuria.

Although histologically, the innermost layer of the urachus is mainly transitional cell, adenocarcinoma is the predominant histological type and most are mucinous. This is probably due to metaplasia arising from chronic inflammation. The treatment of choice for urachal cyst is by complete primary excision. An earlier report suggests a single stage procedure backed with appropriate antibiotic therapy for the treatment of infected UC. However, Yoo et al in their study suggested a 2 stage procedure involving initial incision and drainage, followed by later excision of the urachal remnant. In our case, we adopted a staggered plan of management. Firstly, with administration of broad spectrum antibiotics guided by microbiology sensitivity,and after resolution of sepsis interval primary excision of the cyst, including insertion of a covering corrugated wound drain.

\section{Conclusion}

Urachal anomalies are rare in adults. Presentation is atypical; therefore, a high index of suspicion is required in order to achieve a diagnosis. A triad of lower midline mass, umbilical discharge and sepsis is suggestive, although MRI confirms the diagnosis and defines the surrounding anatomical relationship. Complete surgical excision is the treatment of choice due to the risk of malignant transformation. We recommend a 2 stage treatment with a combination of broad spectrum antibiotics or incision and drainage, followed by interval excision after resolution of sepsis.

\section{Consent}

Written informed consent was obtained from the patient for publication of this case report and accompanying images. A copy of the written consent is available for review by the Editor-inChief of this journal.

\section{References}

1. Hammond G, Yglesias L, Davis JE: The urachus, its anatomy and associated fascia. Anat Rec 1941, 80:271-274.

2. Risher WH, Sardi A, Bolton J: Urachal abnormalities in adults: the Ochsner experience. South Med J 1990, 83:10361039.

3. Begg RC: The Urachus: its Anatomy, Histology and Development. J Anat 1930, 64:170-183.

4. Ashley RA, Inman BA, Routh JC, Rohlinger AL, Husmann DA, Kramer SA: Urachal anomalies: a longitudinal study of urachal remnants in children and adults. $\mathrm{J}$ Urol 2007, 178:1615-1618.

5. Yoo KH, Lee SJ, Chang SG: Treatment of infected urachal cysts. Yonsei Med J 2006, 47:423-427.

6. Flanagan DA, Mellinger JD: Urachalsigmoid fistula in an adult male. Am Surg 1998, 64:762-763.

7. Maruschke M, Kreutzer HJ, Seiter H: Bladder rupture caused by spontaneous 
perforation of an infected urachal cyst. Urologe A 2003, 42:834-839.

8. Ohgaki M, Higuchi A, Chou H, Takashina K, Kawakami S, Fujita Y, Hagiwara A, Yamagishi H: Acute peritonitis caused by intraperitoneal rupture of an infected urachal cyst: report of a case. Surg Today 2003, 33:75-77.

9. Mazzucchelli R, Scarpelli M, Montironi R: Mucinous adenocarcinoma with superficial stromal invasion and villous adenoma of urachal remnants: a case report. J Clin Pathol 2003, 56:465-467.

10. Newman BM, Karp MP, Jewett TC, Cooney DR: Advances in the management of infected urachal cysts. J Pediatr Surg 1986, 21:1051-1054. 